\title{
Current status of sexing mammalian spermatozoa
}

\author{
George E. Seidel, Jr ${ }^{1}$ and Duane L. Garner ${ }^{2}$ \\ ${ }^{1}$ Animal Reproduction and Biotechnology Laboratory, Colorado State University, \\ Fort Collins, CO 80523, USA; and ${ }^{2}$ XY, Inc., Fort Collins, CO 80523, USA
}

\begin{abstract}
Thousands of offspring have now been produced via artificial insemination with spermatozoa sexed by flow cytometry and cell sorting. We are unaware of any other practical approach to sexing spermatozoa that maintains fertility. Accuracy of sexing usually is $85-95 \%$ in most species, but somewhat lower with human spermatozoa. Spermatozoa are sexed in series, one at a time, at routine rates of about 3000 live spermatozoa of each sex per second for most species, and nearly twice that rate under optimal conditions for some species. Owing to various constraints and statistical considerations, there appears to be an upper theoretical limit to sexing spermatozoa of about 10000 live spermatozoa of each sex per second with current methodology. About a quarter of the spermatozoa processed are sexed; the rest are discarded in the process or lost due to logistical constraints. Spermatozoa undergo some damage during sorting, although much less in terms of viability than with routine cryopreservation; fertility is lower with sexed than control spermatozoa. Offspring from sexed spermatozoa appear to have no more abnormalities than do controls, and both groups grow and thrive similarly. Despite high costs and complex procedures, sexing spermatozoa, usually followed by cryopreservation, is being used commercially for cattle and horse production in several countries, and is used to produce girls to avoid X-chromosome-linked genetic diseases.
\end{abstract}

\section{Introduction}

It is now possible to predetermine the sex of offspring from a number of species before fertilization with an accuracy of $85-95 \%$ (Seidel et al., 1999; Welch and Johnson, 1999; Johnson, 2000). This noteworthy accomplishment, first demonstrated convincingly by Johnson et al. (1989), resulted from integration of advances in many fields including chromosomal karyotyping, artificial insemination, maintenance of spermatozoa fertility in vitro, DNAspecific staining, flow cytometry, computer science and high speed cell sorting. Advances from these different disciplines were integrated by a body of innovative scientists co-operating from several sites including Lawrence Livermore National Laboratory, the United States Department of Agriculture Beltsville Agricultural Research Center, Cambridge University, Colorado State University, Atlantic Breeders Cooperative and the company, XY, Inc. (Johnson and Seidel, 1999; Garner, 2001).

The objectives of this review are to explain: (1) why spermatozoa bearing the $\mathrm{X}$ - or $\mathrm{Y}$-chromosome are so similar phenotypically, but what is different between them; (2) the principles and procedures used to sex spermatozoa by total DNA content via flow cytometry and cell sorting; (3) the accuracy, speed, and efficiency of current sperm sexing

Email: gseidel@colostate.edu procedures, particularly explaining why the majority of spermatozoa used are not included in the final sorted product; (4) the extent to which spermatozoa are damaged during current sexing procedures, including effects on fertility, and normality of calves resulting from sexed spermatozoa; and (5) methods other than measuring DNA content, of sexing spermatozoa under recent or current investigation.

\section{Spermatozoa differ in sex chromosome size but in little else}

\section{Historical perspective}

The first documented microscopic identification of sex chromosomes was by Guyer (1910). This observation, along with those of others, generated the idea that mammalian sex might be controlled by these specialized chromatin structures. A variety of techniques, mostly unsuccessful, were used in an attempt to identify which sex chromosome was contained in an individual spermatozoon, with the ultimate goal of separating these gametes. The first convincingly documented difference between mammalian $X$ - and Y-chromosome-bearing spermatozoa was the differential uptake of quinacrine stain by human sex chromosomes (Barlow and Vosa, 1970). The heterochromatin of the Y-chromosome fluoresced more brightly than other chromosomes, including the X-chromosome; however, this 
After centromeres divide, these will form two X-chromosome-bearing spermatozoa

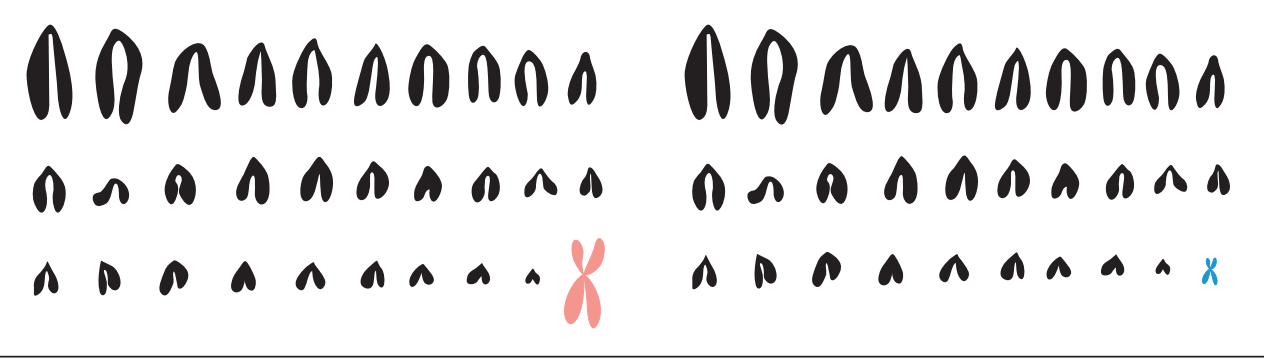

Fig. 1. Karyogram of chromosomes that would be present in two adjacent bovine secondary spermatocytes derived from a primary spermatocyte. The total length of chromosomes of bovine X-chromosome-bearing spermatozoa is about 3.8\% greater than that of Y-chromosome-bearing spermatozoa.

difference did not occur in chromosomes and spermatozoa from non-primates.

\section{0:50 Sex ratios}

Sex is determined in mammals by whether the fertilizing spermatozoon contains an $\mathrm{X}$-chromosome to produce a female or a Y-chromosome to produce a male. As a consequence of the way that chromosomes segregate at meiosis, the chance that a spermatozoon will carry either chromosome is equal. Nature has gone to extremes to minimize phenotypic differences (for example, size, shape, surface properties) between spermatozoa carrying different alleles and different sex chromosomes (Seidel, 1999). Phenotypic equivalence of mammalian spermatozoa within males, despite major allelic differences, is ensured by at least four mechanisms: (1) heterochromatic sex chromosomes are encased in sex vesicles post-meiotically; (2) intercytoplasmic bridges between spermatocytes and spermatids allow interchange of molecules including mRNA; (3) there is limited post-meiotic expression of most autosomal genes during the later stages of spermiogenesis, due in part to extreme condensation of chromatin; and (4) spermatozoa are coated by high-affinity proteinaceous secretions originating from Sertoli cells, excurrent ducts and accessory sex glands, that render the surface of spermatozoa anonymous with regard to possible sex-specific and other allelic differences of the cell membrane (Seidel, 1999).

\section{Karyograms}

Karyograms are displays of chromosomes at metaphase and are used to assess normalcy of numbers and shapes of chromosomes characteristic of a particular species, and to identify sex. For example, a normal male diploid bovine karyogram consists of 58 autosomes plus an $\mathrm{X}$ and a $\mathrm{Y}$ chromosome (all duplicated). A bovine karyogram is illustrated (Fig. 1). The difference in DNA content between $\mathrm{X}$ - and Y-chromosome-bearing bovine spermatozoa is approximately $3.8 \%$; differences for most mammals are in the range 3-4.5\% (Johnson et al., 1987; Johnson, 2000) but in a few species the difference is much larger.

\section{DNA-binding dyes}

The strong binding of certain fluorescent dyes to nucleic acids enables precise quantification of sperm nuclear DNA, in some cases without affecting sperm viability. Many dyes have been used, but only after application of the bisbenzimidazole Hoechst 33342 (2'-(4-ethoxyphenyl)-5(4-methyl-1-piperazinyl)-2', $5^{\prime}$-bi- $1 \mathrm{H}$-benzimidazole $\cdot 3 \mathrm{HCl}$ ) for staining intact spermatozoa was fluorescence quantification of the DNA content of living spermatozoa successful (Johnson et al., 1987). Hoechst $33342(\mathrm{H} 33342)$ is a livecell stain that permeates the cell membrane and binds selectively to A-T base pairs along the minor groove of dsDNA. It is a yellow solid having a formula weight of 561.9 $\left(\mathrm{C}_{27} \mathrm{H}_{28} \mathrm{~N}_{6} \mathrm{O} \cdot 3 \mathrm{HCl}\right)$ and is moderately water-soluble and relatively non-toxic. $\mathrm{H} 33342$ usually is excited with the 351 or $364 \mathrm{~nm}$ lines of an argon-ion laser or other sources of fluorescence excitation such as mercury lamps and exhibits a relatively large Stokes shift (excitation/emission maxima of about 350/460 nm), making it very useful in assessing precise amounts of DNA in cells.

Most DNA stains intercalate between the base pairs of the DNA, thereby presumably increasing their mutagenicity. However, H33342 is not an intercalative dye, which probably makes it safer to use (Watkins et al., 1996). Nevertheless, $\mathrm{H} 33342$ may be toxic to workers at high doses, so it must be used carefully. Binding of $\mathrm{H} 33342$ to DNA is stabilized by a combination of hydrogen bonding, van der Waals forces, and electrostatic interactions between the dye molecule and the negatively charged DNA molecule. The strength of this multiple-binding interaction may contribute to the putative radioprotective properties of the $\mathrm{H} 33342$ molecule (Young and Hill, 1989).

Dead or moribund cells in the population of spermatozoa stained with $\mathrm{H} 33342$ can be identified by adding propidium iodide (Johnson et al., 1994). More recently, this classical dead-cell stain has been replaced with red 
(a) Overview of sperm sorter; computer not shown.

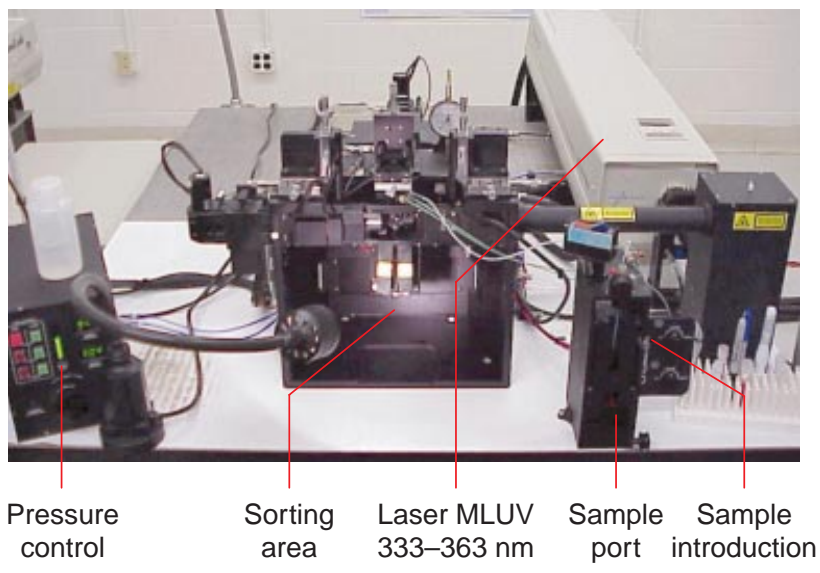

(b) Optics and hydrodynamics

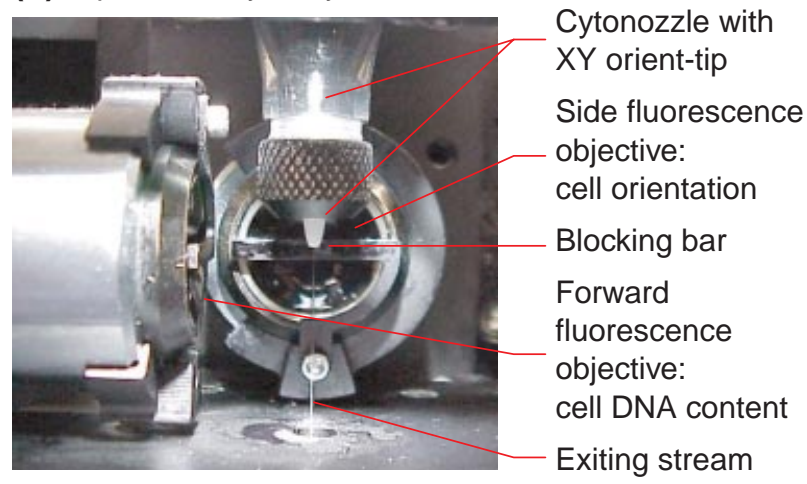

This figure shows the objectives for the forward and side fluorescence detectors, Cytonozzle with orienting tip, and blocking bars to keep the incidental light from the beam from reaching the detectors. Forward fluorescence measures DNA content and side fluorescence measures cell orientation of spermatozoa in the exiting stream.

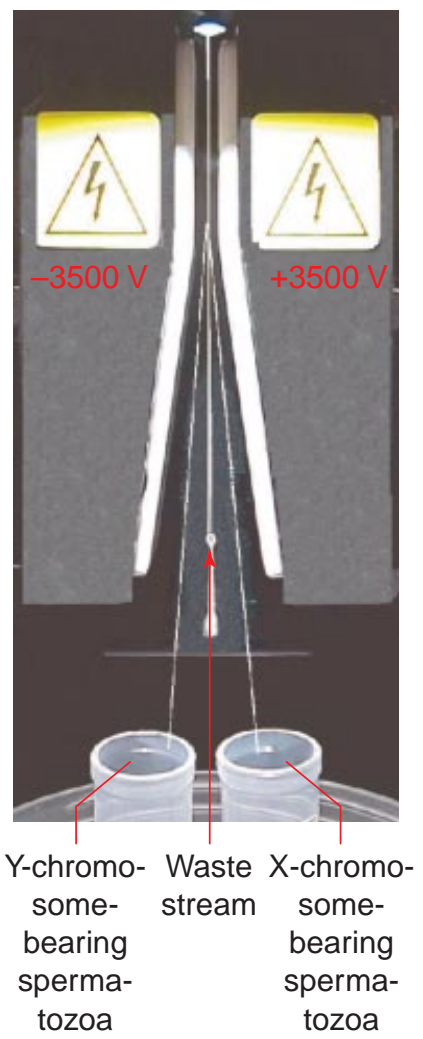

(d) Both X- and Y-chromosome-bearing spermatozoa can be sorted simultaneously. Droplets containing Y-chromosome-bearing spermatozoa are given a positive charge, so are attracted to the negative field to the left whereas droplets containing X-chromosomebearing spermatozoa are given a negative charge and are attracted to the right.

The respective streams of droplets are collected in separate test tubes that contain an egg yolk medium to keep spermatozoa healthy.

Droplets with no spermatozoa, dead spermatozoa, or unsexable spermatozoa are uncharged, and drop straight into the waste stream.

Fig. 2. MoFlo SX spermatozoa sorter.

food dye (FD\&C40) to avoid potential mutagenic effects of propidium iodide (Johnson and Welch, 1999; Schenk et al., 1999). Other food dyes also are effective. The mechanism of action is to quench the H33342 fluorescence of spermatozoa that have damaged membranes so that they can be removed during the sorting process by dead-cell gating.

\section{Separation of spermatozoa by DNA content via flow cytometry and cell sorting}

Detailed procedures for flow-sorting mammalian spermatozoa are beyond the scope of this review, and depend on the species and application. However, an overview will be provided. 


\section{Box 1. Steps in sexing spermatozoa}

The following steps summarize the overall process for sexing bull spermatozoa. More details are given by Schenk et al. (1999).

(1) Collect semen.

(2) Store up to $9 \mathrm{~h}$ undiluted at $20-23^{\circ} \mathrm{C}$ until staining.

(3) Dilute an aliquot in staining medium to $200 \times 10^{6}$ spermatozoa $\mathrm{ml}^{-1}$.

(4) Stain aliquot with $\mathrm{H} 33342$ for $45 \mathrm{~min}$ at $34^{\circ} \mathrm{C}$.

(5) Dilute in sorting medium to $100 \times 10^{6}$ spermatozoa $\mathrm{ml}^{-1}$; add food colouring.

(6) Sort batch for $90 \mathrm{~min}$.

(7) Repeat steps 3-6 every 90 min for up to $9 \mathrm{~h}$ from the same semen sample.

(8) Concentrate sample to approximately $40 \times 10^{6}$ spermatozoa $\mathrm{ml}^{-1}$ by centrifugation at $850 \mathrm{~g}$ for $20 \mathrm{~min}$ and discard the supernatant. Sorted spermatozoa are diluted (to approximately $8 \times 10^{5}$ spermatozoa $\mathrm{ml}^{-1}$ ) as a consequence of mixing sorting medium, catch fluid and sheath fluid (Fig. 2d).

(9) Accumulate several sort batches and pool.

\section{Steps applied to semen during sorting}

The process used by XY, Inc. for sorting bull spermatozoa (see Box 1) will be used as a framework for this section. For nearly all applications, freshly collected semen is used. Spermatozoa that have been cryopreserved and thawed do not tolerate the sexing process well, particularly if they are to be re-frozen. Since sperm sorting is a slow process relative to the needs for most applications, keeping spermatozoa healthy between semen collection and sorting is important. Schenk et al. (1999) have done several studies on this step with bull semen, and of the many procedures tried, storing the semen undiluted at $20-23^{\circ} \mathrm{C}$ for $0-8 \mathrm{~h}$ was the best procedure. Storage of bovine spermatozoa for longer than $8 \mathrm{~h}$ has not been studied thoroughly in the context of flow-sorting, although limited studies with various methods have shown that dilution during storage has adverse effects on H33342 DNA-staining properties. On the other hand, stallion spermatozoa are sexed routinely after storage in appropriate diluents at $5-15^{\circ} \mathrm{C}$ for $18 \mathrm{~h}$, resulting in excellent fertility if spermatozoa are not cryopreserved after sorting. Logistics dictate the staining of a new batch of bovine spermatozoa from the raw semen every $1-2 \mathrm{~h}$, thus minimizing the time of exposure of spermatozoa to high concentrations of dye, and minimizing the destaining that occurs during dilution between staining and sorting.

In subsequent steps of the sorting process (Box 1), spermatozoa reside in a number of fluids: staining medium; sorting medium; a mixture of catch fluid, sheath fluid and sorting medium; and fluids for preparation of the final product after concentration by centrifugation, for example, cryoprotectant media. The medium for staining spermato- zoa with H33342 is a modified Tyrode's albumin lactate pyruvate (TALP) (Schenk et al., 1999). This is diluted with TALP containing egg yolk to which vital-staining food dye is added for sorting. During the sorting process, spermatozoa in the sorting medium are mixed with Tris-based sheath fluid containing citric acid and fructose. Sheath fluid functions as a cylindrical wall of fluid surrounding the core stream of sorting medium (containing the spermatozoa) that guides the flow of fluid through the flow cytometer. The exiting fluid is composed of approximately $90 \%$ sheath fluid and $10 \%$ core stream fluid. After spermatozoa pass through the sorter, the streams of $\mathrm{X}$ - and $\mathrm{Y}$-chromosome-bearing spermatozoa are collected into tubes containing a $22 \%$ egg yolk-Tris extender (catch fluid; Schenk et al., 1999). As the test tube fills during sorting (Fig. 2d), the initial catch fluid is continually diluted by the stream exiting the sorter nozzle. The sorting, sheath and catch fluids obviously need to be compatible with maintaining sperm fertility, but respective fluids also must have certain electrical and viscosity properties, as well as not interfere with staining and fluorescence.

Spermatozoa that accumulate every $3-4 \mathrm{~h}$ are frozen as a batch, so there are usually two freezing batches for each ejaculate, each freezing batch consisting of several sorting batches. In many instances, two or three sorters (up to ten at one location) are used simultaneously for the same ejaculate to improve the scale of operation. This procedure enables freezing of reasonable numbers of doses of spermatozoa per batch every 3-4 h, and minimizes the time between sorting and cryopreservation of spermatozoa.

\section{Principles of flow cytometer and cell sorter use for sexing spermatozoa}

Components of a flow cytometer and cell sorter designed for sexing spermatozoa are illustrated (Fig. 2). In brief, as a spermatozoon passes two fluorescence detectors at $90^{\circ}$ angles to each other (Fig. 2b), each detector measures the intensity of fluorescence resulting from excitation of the DNA-bound dye molecules by light, which usually is generated by a laser. The wavelengths of light used depend on the light source, and if and how the light is filtered. Importantly, if, for example, an argon laser is used as a light source, spermatozoa are not exposed to the damaging low ultraviolet wavelengths that are absorbed by nucleic acids and proteins.

The strength of the fluorescence signals obviously depends on the number of fluorescing molecules bound to DNA. This is the basis for sexing spermatozoa. In addition, the signal depends on a number of other parameters, including the laser intensity (Guthrie et al., 2002), whether and how the laser is pulsed, optical properties of the entire system, sensitivity of detectors and electronic noise. All of these factors must be kept as constant as possible to resolve the small differences in DNA content between $X$ - and Y-chromosome-bearing spermatozoa.

The main obstacle to accurate quantification of sper- 
matozoa DNA with this approach is the geometry of the sperm head, which is paddle-shaped in most species of interest. The intensity of fluorescence is lowest if the flat face of the paddle is oriented toward a detector, and highest when the edge is so oriented. Flat orientation results in the most accurate discrimination between $X$ - and $Y$ chromosome-bearing spermatozoa, so only spermatozoa oriented in this way are sorted (Fig. 2b). The second detector at $90^{\circ}$ to the laser is used to diagnose orientation (Fig. 2b). As the fluorescence signal is highest for spermatozoa oriented with their paddle edge toward this $90^{\circ}$ detector, only the population of spermatozoa that emit peak fluorescence to the $90^{\circ}$ detector are considered oriented appropriately for sexing by the contemporaneous signal to the $0^{\circ}$ detector (Fig. 2b).

Since sperm orientation will be random with respect to detectors in a cylindrical stream of fluid, only about $10 \%$ of spermatozoa will be sufficiently well oriented for accurate DNA measurements under those circumstances. A considerable effort, therefore, has been made to increase this percentage by modifying the cylindrical geometry of the fluid stream (Johnson and Welch, 1999; Rens et al., 1998, 1999). Although details will not be reviewed here, about $70 \%$ of spermatozoa are oriented correctly with current technology (Johnson and Welch, 1999) (Fig. 2b). Other approaches to this problem, such as measuring total fluorescence with spherical detectors (Sharpe et al., 1997) have not yet become useful.

\section{How fast can high-speed flow cytometers and cell sorters sex spermatozoa?}

Modern flow cytometers sort spermatozoa at rates that are commercially viable by propelling spermatozoa through the system at accelerating speeds approaching $90 \mathrm{~km} \mathrm{~h}^{-1}$ when they exit the nozzle. Discrete fluorescence signals from two detectors at $90^{\circ}$ angles to each other are produced at a rate of over 180000 measurements per detector per second, and the information is processed by computer and relayed to the stream-charging mechanism by the time that the spermatozoa have travelled a few $\mathrm{cm}$, such that the droplets to be formed with $\mathrm{X}$-chromosome-bearing spermatozoa have different electrical charges from those with Y-chromosome-bearing spermatozoa. Computation is so fast and sensitive that should a droplet contain two $X$ - or Y-chromosome-bearing spermatozoa by chance, even these droplets can be saved, while droplets with dead or misoriented spermatozoa can be discarded.

Sorting of nearly all non-human spermatozoa for sex currently is done with the high pressure, high speed, multipurpose flow cytometer built by Cytomation, Inc. (Fort Collins, CO), model MoFlo ${ }^{\circledR}$ SX equipped with an argon laser, detectors for fluorescence, and a special spermatozoa orientation nozzle modified from one developed by Rens et al. (1998, 1999). Detailed technical specifications are beyond the scope of this review, but a key parameter is flow rate past the detectors, which is over $20 \mathrm{~m} \mathrm{~s}^{-1}$. Theoretically, as spermatozoa are about $100 \mu \mathrm{m}$ in length, if they were perfectly oriented in a column head-to-tail, about 200000 spermatozoa could pass the detector each second, and even more if the size of sperm heads only were considered. Unfortunately, the distribution of spermatozoa in such a column approximates a Poisson distribution. This means that there is considerable space between each flowing spermatozoon. If the concentration of spermatozoa is increased, while this average space shortens, more and more spermatozoa are relatively clumped, so that for practical purposes, fluorescence signals between them are too close together for the system to process them separately, resulting in more and more unusable coincidence signals. A second, related problem is that some spermatozoa are statistically 'between droplets', so it is unclear in which droplet (Fig. 2c) the spermatozoa will reside; in this case, both drops are discarded (termed an abort). Theoretically and empirically, the optimum throughput in this statistical situation is achieved at about 25000 spermatozoa per second, the so-called event rate. Event rates can be changed by modifying the concentration of spermatozoa in the sample, either directly or by changing the ratio of the core stream to sheath fluid. However, there is no net benefit above about 25000 spermatozoa per second due to increasing rates of abortion or coincidence. Another option is to increase the flow rate past the detector by increasing the pressure of the entire system. However, this similarly results in increased rates of abortion or coincidence and damage to the spermatozoa.

Superimposed on the above considerations are physical constraints of droplet size. Droplets are formed as the column of fluid exits the nozzle due to vibrations set up by a piezoelectric mechanism in the column, even before the spermatozoa pass the detectors. The frequency of vibrations must be matched with characteristics of the nozzle tip, particularly diameter, as well as viscosity of the fluid and system pressure. Roughly, with a nozzle with an internal diameter of $70 \mu \mathrm{m}$, nearly 70000 droplets can be formed per second at pressures of 40 psi, but fewer at lower pressures and larger orifice diameters. The ideal situation is to make as many droplets per unit time as possible without disturbing other properties of the system such as efficacy of sperm orientation.

Matching a Poisson distribution of 25000 spermatozoa per second in the column of fluid to discrete droplets of a specific size produced at 70000 per second occurs imperfectly. About $25 \%$ of droplets will contain a single spermatozoon, a few per cent will contain two or more spermatozoa, and $>70 \%$ will not contain any spermatozoa. There is no simple way around these inefficiencies as they are inherent due to laws of statistics and physics. They place an upper limit on sorter performance at approximately 80000 droplets per second at 50 psi and sorting about 10000 live spermatozoa of each sex per second per nozzle, assuming perfect sperm orientation and perfect resolution of oriented spermatozoa. A better option may be lower pressures and fewer droplets, resulting in less sperm 


\section{Box 2. Typical efficiency of sexing spermatozoa}

Aliquot of stained spermatozoa (100\%)

(1) Residual loss of spermatozoa in staining tube $10 \%$

(2) Losses in sorter tubing between batches, between males and to prevent or correct plugged nozzles

Spermatozoa that are evaluated (78\%)

(3) Spermatozoa discarded due to malorientation $30 \%$

(4) Spermatozoa discarded due to coincidence $15 \%$

(5) Discarded dead spermatozoa $\quad 10 \%$

Potentially sortable spermatozoa (35\%)

(6) Spermatozoa discarded to maintain purity $12+\%$ (Fig. 3) because distributions of $X$ - and Y-chromosome-bearing spermatozoa fluorescence overlap

(7) Probable aneuploid spermatozoa discarded (Fig. 3)

(8) Spermatozoa discarded due to aborts and droplets with both $\mathrm{X}$ - and Y-chromosomebearing spermatozoa

Spermatozoa that have been sorted (30\%)

(9) Spermatozoa lost because of spraying (missing the fluid in the bottom of the collection tube Fig. 2d)

(10) Losses of spermatozoa in the supernatant after centrifugation (concentration step)

(11) Loss of spermatozoa during filling and sealing straws including incomplete volume of residue in the last straw

Sorted spermatozoa that are frozen $(23 \%)$

(12) Use of spermatozoa for quality control (accuracy and motility after thawing) Spermatozoa available for insemination (22\%; $11 \%$ of each sex at $90 \%$ accuracy)

Percentage values in the right column refer to percentages of the respective sub-headings rather than of the starting material.

damage but slower sorting speeds. To circumvent these limits would require fundamental changes in sorting procedures, changes that seem unlikely to be developed over the next few years.

\section{Efficiency of sperm sorting}

There is potential for sperm losses at virtually every step of processing and sorting (see Box 2). The losses are highly dependent on the staining properties of a particular ejaculate as well as on the skill and the care taken by the technicians doing the work. Losses are multiplicative. Typical cumulative efficiency for the 12 sequential steps in Box 2 at $90 \%$ accuracy of sorting is $22 \%$ (11\% of each sex). Of course, $10 \%$ of the spermatozoa were dead and were purposely discarded.
The above efficiencies vary as a result of the quality of the sample and the speed of sperm flow through the system. Sometimes ejaculates degrade during the day and the efficiencies listed become much lower, particularly at step 1, but also at steps 5 and 6 . About $5 \%$ of the time, the entire day's work is discarded because the motility of spermatozoa after thawing does not meet quality control standards, as also occurs with unsexed spermatozoa. As fewer sexed spermatozoa usually are packaged per insemination dose than conventionally, these losses do not necessarily lead to fewer inseminates per volume of semen used than occur with normal semen processing with standard numbers of spermatozoa.

Under most practical circumstances, only part of an ejaculate will be sorted because the process is slow relative to the number of spermatozoa available. As spermatozoa will undergo various stresses during the sorting process, fresh samples seem to tolerate sorting significantly better than gametes that have been stored for hours. The above considerations, plus the number of sperm sorters available will determine how many spermatozoa in an ejaculate should be held for sorting, and how many will be available for non-sorted applications.

The raw data from flow cytometers can be displayed in many ways. The simplest way is to present only the fluorescence data from the $0^{\circ}$ detector for adequately oriented, live spermatozoa (Fig. 3), that is those showing maximal fluorescence with the $90^{\circ}$ detector (see Johnson and Welch, 1999). Misoriented and dead spermatozoa are not considered in Fig. 3; droplets containing them and spermatozoa not resolvable due to coincidence would receive no electric charge when exiting the nozzle of the sorter and, thus, would be discarded in the waste stream (Fig. 2d). Thus, the fluorescence from the $0^{\circ}$ detector of the oriented, live subset of resolvable spermatozoa (less than half of the spermatozoa monitored) can be plotted as in Fig. 3. Note that the distributions of fluorescence of the $\mathrm{X}$ - and $\mathrm{Y}$-chromosome-bearing spermatozoa overlap, resulting in an overall bimodal distribution. By discarding the spermatozoa in the centre of the bimodal distribution (shaded in Fig. 3), the spermatozoa to the left are primarily Y-chromosome-bearing spermatozoa and those to the right are primarily X-chromosome-bearing spermatozoa. The width of the shaded area in Fig. 3 generally is set to produce spermatozoa sexed at $90 \%$ accuracy. Spermatozoa in the extreme tails of the curve often have missing (left tail) or extra chromosomes (right tail); these aneuploid spermatozoa also are discarded by not charging droplets containing them.

\section{Damage to spermatozoa and normality of calves}

\section{Damage to spermatozoa during sorting}

Fertility of sorted spermatozoa is somewhat lower than that of controls (Seidel et al., 1999; Buchanan et al., 2000) as is survival of sorted spermatozoa after cryopreservation 


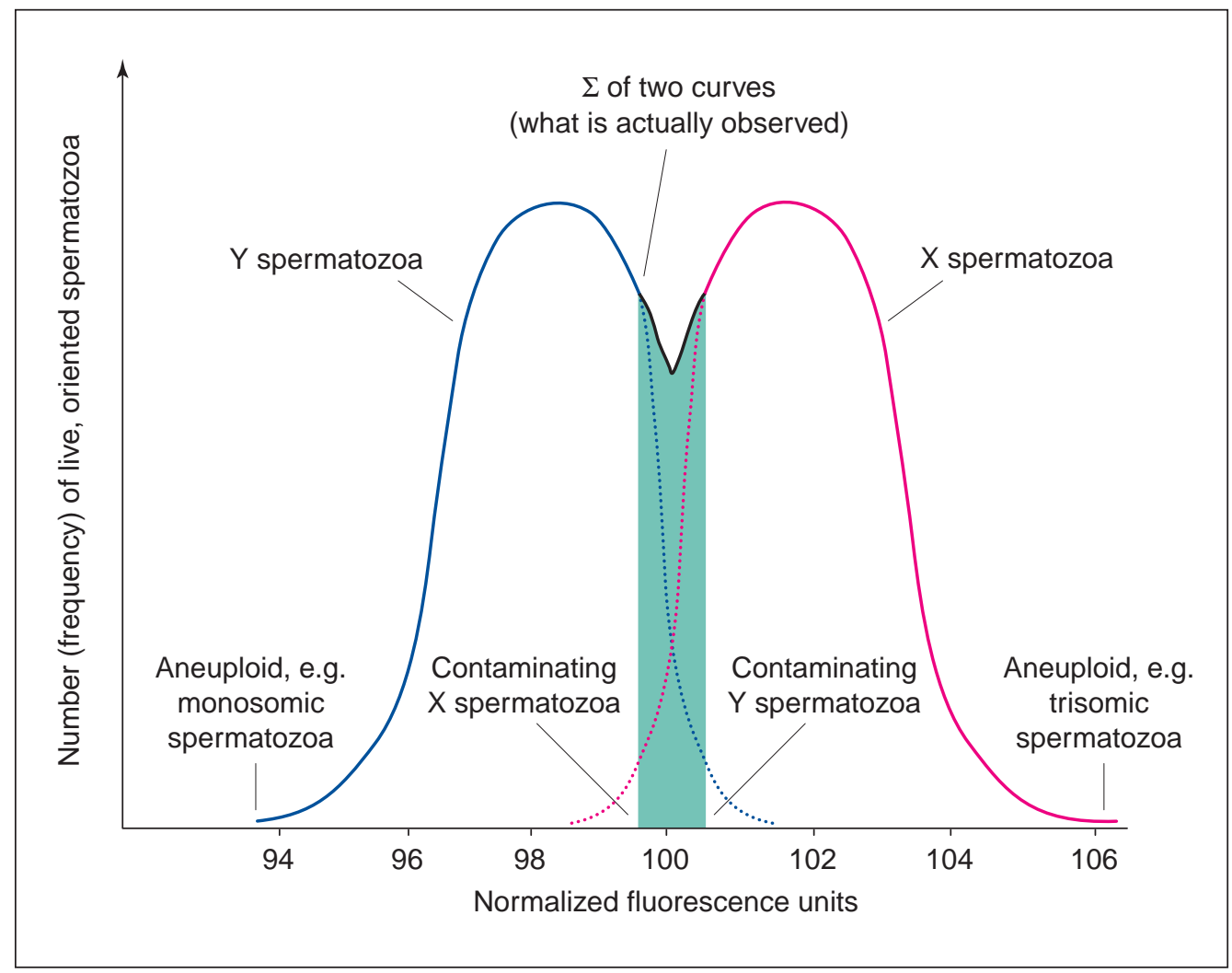

Fig. 3. Theoretical histograms illustrating sorting efficiencies of $X$ - and $Y$-chromosome-bearing bovine spermatozoa recovered from the sorter. The initial sample would contain 50\% Y- (blue) and 50\% $\mathrm{X}$-chromosome-bearing (pink) spermatozoa. The shaded area between the $\mathrm{X}$ - and $\mathrm{Y}$-chromosome-bearing spermatozoa peaks show magnitude of the overlap, where it is impossible to distinguish between spermatozoa carrying an X-chromosome and those with a Y-chromosome. These spermatozoa are discarded. The wider the shaded area, the purer the $X$ - and $Y$-chromosome-bearing spermatozoa populations will be, but the more spermatozoa that will be discarded.

(Schenk et al., 1999). Unfortunately, most studies on fertility with sexed spermatozoa are confounded by using fewer sexed spermatozoa per insemination dose than normal procedures would dictate. When similar numbers of spermatozoa per dose have been used, pregnancy rates with sexed spermatozoa usually have been $60-80 \%$ of unsexed control spermatozoa (Doyle et al., 1999; Seidel et al., 1999). Pregnancy losses in cattle between 1 and 2 months of gestation have been $1-2 \%$ higher with low insemination doses of sexed spermatozoa than with normal insemination doses of unsexed spermatozoa (Seidel et al., 1999). It will take large numbers of animals to determine whether this is a true effect, or whether this non-significant difference will dissipate with more thorough study.

One recent study concerns the amount of damage to DNA and mortality of spermatozoa subjected to various combinations of mechanical forces at $50 \mathrm{psi}$, exposure to laser, and staining procedures during sorting (Fig. 4; Garner et al., 2001). Sorted spermatozoa were further analysed by flow cytometry for both DNA integrity (Evenson, 1989) and failure to exclude propidium iodide as a measure of dead spermatozoa. It is clear (Fig. 4) that most of the damage resulted simply from processing the spermatozoa through the sorter, even with no staining and no exposure to laser light. Additional damage due to exposure to laser and dye was small and not statistically significant, in agreement with other studies (Libbus et al., 1987; Guthrie et al., 2002). Very recent studies from our laboratory (Suh and Schenk, in press) indicate that much of the mechanical damage noted can be alleviated by lowering the pressure of the fluid during sorting. This is likely to improve fertility of sexed spermatozoa compared with studies to date in which spermatozoa have been sorted at $50 \mathrm{psi}$.

\section{Normalcy of offspring of sex-sorted spermatozoa}

Several thousand offspring from seven mammalian species (cattle, pigs, rabbits, horses, sheep, elk and humans), primarily cattle, have been produced after $\mathrm{H} 33342$-staining and flow-sorting of the fertilizing spermatozoa. No gross 


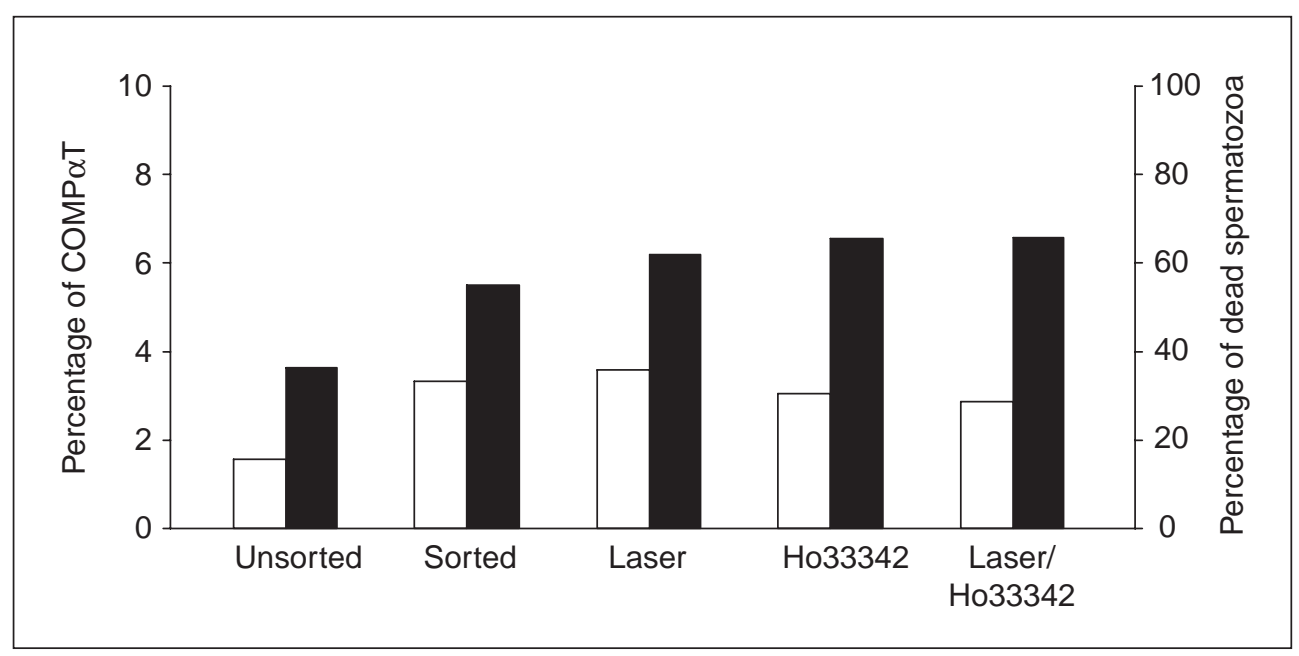

Fig. 4. Percentages of $(\boldsymbol{\square})$ dead spermatozoa and $(\square)$ spermatozoa with damaged DNA after thawing as determined by the spermatozoa chromatin stability assay (percentage of COMP $\alpha \mathrm{T}$, cells outside of the main population) after (1) unsorted control, (2) passing spermatozoa through the sorter without laser or staining, (3) with laser but no staining, (4) with staining, but no laser, and (5) with both staining and laser (modified from Garner et al., 2001).

abnormalities have been reported (Morrell and Dresser, 1989; Johnson, 1995; Catt et al., 1997; Doyle et al., 1999; Fugger, 1999; Johnson and Welch, 1999; Seidel et al., 1999), although we are aware of an anecdotal report of one abnormal calf. These data indicate that the DNA of flowsorted spermatozoa that result in offspring is not severely damaged. Here, we report preliminary results from a large, ongoing study.

Black Angus heifers were inseminated with frozen, sexed and unsexed control spermatozoa during 3 days. Standard sexing procedures were used (Schenk et al., 1999; Seidel et al., 1999). Inseminations were balanced over semen from two bulls, four inseminators, two doses of sexed spermatozoa $\left(1.5 \times 10^{6}\right.$ and $4.5 \times 10^{6}$ frozen spermatozoa per insemination dose) and control $\left(20 \times 10^{6}\right.$ frozen, unsexed spermatozoa), and two insemination times (12 and $24 \mathrm{~h}$ after oestrus). However, all inseminations were carried out 'blind' with respect to treatments. Some heifers were inseminated with $\mathrm{X}$-chromosome-bearing spermatozoa, and others with Y-chromosome-bearing spermatozoa, depending on pedigrees and perceived commercial value of the resulting calves. Detailed fertility results will be reported elsewhere; briefly, there was no significant difference in 2 month pregnancy rates determined by ultrasonography between the two doses of sexed spermatozoa (overall average, 53\%; $n=245)$, which, however, were lower $(P<0.05)$ than control pregnancy rates $(66 \% ; n=126)$.

The pregnant heifers from this study were then allocated to three different farms and managed by routine husbandry procedures, but with different intensity of management. Personnel managing the cattle were unaware of which heifers were pregnant with sexed or control spermatozoa. Between 2 months of pregnancy and term, there were four abortions of $86(4.7 \%)$ control pregnancies and seven abortions of 130 (5.4\%) pregnancies from sexed spermatozoa, a very small, non-significant difference.

There were no significant differences in rates of neonatal death or accumulated deaths to weaning between calves derived from sorted versus control spermatozoa, nor were there any treatment effects on duration of gestation, birth weight or weaning weight (Table 1). No congenital abnormalities were observed. The only significant effects $(P<0.01)$ were that male calves were heavier at birth and weaning than female calves. Therefore, there appear to be no detrimental effects of sorting spermatozoa on resulting offspring. There were large differences in the survival of calves among the three farms, with deaths to weaning ranging from 5 to $18 \%$. Such routinely observed differences in husbandry from farm to farm obviously are much greater than any effects of sexing spermatozoa. Although this data set resulting from 371 inseminations has a respectable sample size (123 calves from sorted sperm; 82 from controls), more extensive data need to be examined to be certain that there is no small increase in problems of offspring resulting from sorted spermatozoa. In addition, the fate of the minute quantity of $\mathrm{H} 33342$ in the female reproductive tract from the spermatozoa that do not fertilize oocytes probably merits further study.

\section{Methods for sexing spermatozoa other than DNA quantification with DNA binding dyes}

In addition to fluorescence flow cytometry and cell sorting based on DNA content, separation of spermatozoa containing the X-chromosome from those with the Y-chromosome has been attempted with a variety of other techniques 
Table 1. Characteristics of calves produced from frozen flow-sorted and control spermatozoa

\begin{tabular}{|c|c|c|c|c|c|c|c|}
\hline Treatment & $\begin{array}{l}\text { Sex of calf } \\
\text { at birth }\end{array}$ & $n$ & $\begin{array}{l}\text { Mean duration } \\
\text { of gestation } \\
\text { (days) } \pm \text { SE }\end{array}$ & $\begin{array}{c}\text { Number of } \\
\text { calves that died } \\
\text { neonatally }\end{array}$ & $\begin{array}{c}\text { Mean } \\
\text { birth weight } \\
(\mathrm{kg}) \pm \mathrm{SE}^{\mathrm{b}}\end{array}$ & $\begin{array}{c}\text { Number of } \\
\text { calves that died } \\
\text { up to weaning }\end{array}$ & $\begin{array}{c}\text { Mean weaning } \\
\text { weight }(\mathrm{kg}) \pm \mathrm{SE}^{\mathrm{b}}\end{array}$ \\
\hline Sorted ${ }^{\mathrm{a}}$ & Male & 59 & $281.0 \pm 0.72$ & $2(3.4 \%)$ & $33.5 \pm 0.72$ & $3(5.1 \%)$ & $267 \pm 3.4$ \\
\hline Sorted ${ }^{\mathrm{a}}$ & Female & 64 & $280.3 \pm 0.65$ & $6(9.4 \%)$ & $30.6 \pm 0.63$ & $8(12.5 \%)$ & $259 \pm 3.8$ \\
\hline Control & Male & 48 & $280.1 \pm 0.61$ & $4(8.3 \%)$ & $34.4 \pm 0.74$ & $8(16.7 \%)$ & $267 \pm 4.8$ \\
\hline Control & Female & 34 & $280.8 \pm 0.70$ & $3(8.8 \%)$ & $31.2 \pm 0.87$ & $3(8.8 \%)$ & $261 \pm 5.9$ \\
\hline
\end{tabular}

aAccuracy of sorting was 56 of 59 males (95\%) produced with Y-sorted spermatozoa, and 62 of 64 females (97\%) produced with X-sorted spermatozoa.

bThe only significant differences among treatments were that male calves were heavier than female calves $(P<0.01)$ at birth and weaning.

(Amann and Seidel, 1982). A brief update of recent and ongoing work with these technologies follows.

\section{Gradient swim-down procedure}

Successful separation of $\mathrm{X}$ - and Y-chromosome-bearing human spermatozoa using an albumin gradient was first reported by Ericsson et al. (1973). The conceptual basis for this method is that $\mathrm{Y}$-chromosome-bearing spermatozoa are smaller in size and exhibit a greater downward swimming velocity than $\mathrm{X}$-chromosome-bearing spermatozoa within vertical columns of high density human serum albumin (Ericsson et al., 1973). A fraction enriched with Ychromosome-bearing spermatozoa can be obtained by harvesting the first $22 \%$ of spermatozoa to swim to the bottom of the gradient, and discarding the remainder (Ericsson and Ericsson, 1999). Ericsson and Ericsson (1999) reported that the latest version of this technique increased the percentage of male children born to $70-80 \%$. However, the validity of sex pre-selection by this approach has been challenged repeatedly (for example, Evans et al., 1975). This technique has never been shown to sex spermatozoa accurately from mammals other than humans (Beal et al., 1984; White et al., 1984). Furthermore, it is not possible ethically to do prospective, randomized, 'blind' trials with sexed human spermatozoa rigorously to document the true efficacy of this technique. Another interesting aspect of the use of this method is that when women are treated with clomiphene citrate to induce ovulation before insemination, the sex ratio is reversed, so that up to $73 \%$ females are born (Ericsson and Ericsson, 1999).

\section{Surface antigenic differences}

Various immunological approaches to sexing spermatozoa of mammals have been tested without repeatable success (Hoppe and Koo, 1984; Hendriksen et al., 1996; Hendriksen, 1999). One approach was to target $\mathrm{H}-\mathrm{Y}$ antigen. However this molecule appears to be on both $\mathrm{X}$ - and Y-chromosome-bearing spermatozoa (Hoppe and Koo, 1984) and is possibly derived from Sertoli cells. An immunological approach, however, would be highly desirable because inexpensive batch processing could be used to enrich for either $\mathrm{X}$ - or $\mathrm{Y}$-chromosome-bearing spermatozoa. Howes et al. (1997) suggested that their inability to detect sex-specific differences in spermatozoa surface antigenicity using rigorous biochemical methods indicates that an immunological approach to semen sexing was unlikely to work. Nonetheless, attempts using this approach recur regularly.

A recent report suggests that a viable immunological sperm sexing procedure can be developed using a more rigorous method to isolate sex-specific proteins (SSPs) (Blecher et al., 1999). In this newer approach, non-SSPs were removed immunologically before the attempted isolation of SSPs because they are likely to be more highly conserved than non-SSPs. Antibodies to SSPs were raised and used to identify SSPs by affinity chromatography (Blecher et al., 1999). Antibodies to purified female fetal SSPs caused agglutination of approximately half of the bovine spermatozoa and when the unagglutinated spermatozoa were isolated and used in bovine IVF, they produced $>90 \%$ male embryos (Blecher et al., 1999). This immunological approach, which implies post-meiotic transcription or translation of SSPs that do not equilibrate through inter-spermatid cytoplasmic bridges, appeared promising. However, to date there are no reports of producing sex-selected offspring with this procedure despite considerable investment of resources over several years.

\section{Free-flow electrophoresis}

Electrophoretic separation of mammalian sex determining spermatozoa has been attempted by many investigators without significant success (Kiddy and Hafs, 1971; Mohri et al., 1987). This approach, which is based on the possibility that the electric charge on the surface of $X$-chromosome-bearing spermatozoa differs from that exhibited by Y-chromosome-bearing spermatozoa, uses an electric field to separate spermatozoa into the two major classes (Kaneko et al., 1984). Spermatozoa are introduced into the free-flow apparatus continuously and move along different paths to be collected as two main fractions. F-body examination of separated human spermatozoa using quinacrine staining indicated that the purported X-chromosomebearing fraction was relatively pure, but that the spermatozoa in the $\mathrm{Y}$-chromosome-bearing fraction were not (Kaneko et al., 1984). However, the resulting spermatozoa were compromised because sperm motility was reduced 
significantly. Successful separation of sex-determining spermatozoa of other mammalian species has not been reported, although this approach may currently be undergoing reappraisal.

\section{Sperm sorting based on volumetric differences}

Spermatozoa containing an $X$ chromosome are theoretically larger than those containing a $\mathrm{Y}$ chromosome. van Munster et al. (1999a) recently used interference microscopy and subsequent image analysis to demonstrate a difference in sperm head volume that matched differences in DNA content between X- and Y-chromosome-bearing bovine spermatozoa. A method based on this principle has been developed for sorting live spermatozoa by using interference microscopy optics with a flow cytometer (van Munster, 2002). Such a method, which eliminates the need to use DNA-specific dyes, would be a highly attractive alternative method for sexing mammalian spermatozoa. Unfortunately, the potential purity of spermatozoa separated using volumetric measurements cannot exceed $80 \%$ purity of either sex based on theoretical considerations (van Munster et al., 1999b), and recent efforts to make this practical have not been encouraging (van Munster, 2002).

\section{Centrifugal countercurrent distribution}

Recently, Ollero et al. (2000) have attempted to sex ram spermatozoa by centrifugal countercurrent distribution using an aqueous two-phase system. This is a chromatographic process that partitions cells into a stationary, lower phase and a mobile, upper phase, repeated numerous times. Centrifugation was used to speed the partitioning process, so a set of 59 partitions was done in about $1 \mathrm{~h}$.

Ollero et al. (2000) found that they could obtain fractions of up to $75 \%$ Y-chromosome-bearing spermatozoa with reasonable viability using this procedure at certain salt concentrations. However, they presented no data on repeatability of the process or fertility of the spermatozoa. Each batch produced about $6 \times 10^{6}$ spermatozoa $(75 \%$ Ychromosome-bearing). This procedure needs to be verified; it also may not be as successful for species with less difference in DNA content between X- and Y-chromosomebearing spermatozoa than in sheep (4.2\%; Johnson, 1995).

\section{Genetic approaches}

Some years ago, a genetic approach to sperm sexing was suggested (Seidel, 1988), which subsequently has been demonstrated (Herrmann et al., 1999). This approach involves the well known transmission distortion ratio of alleles at the T loci on chromosome 17 in mice. Rather than having half of the offspring with each of two alleles, > 90\% (the percentage depends on the specific allele and genetic background) receive the detrimental allele, a violation of Mendel's law of independent assortment. Basically, spermatids with one allele 'poison' those with the other allele, presumably via intracellular bridges in spermatids. Herrmann et al. (1999) demonstrated this concept by placing part of this genetic system on the $\mathrm{Y}$ chromosome using transgenic procedures. They produced a strain of mice that produced $66 \%$ males (217/331) in the course of natural mating. In principle, this could be done in any species, with either sex chromosome; however, such a project would be expensive and complicated, and there are some caveats in non-murine species.

\section{Perspectives on commercialization}

Commercialization of sperm sexing has begun for cattle and is imminent for horses (Buchanan et al., 2000), and the methodology is being used on a limited scale to produce human babies, particularly to produce girls to avoid Xlinked genetic disease (Johnson et al., 1993; Fugger et al., 1998). This review has focused on the actual process of sexing spermatozoa by flow cytometry and cell sorting, and illustrates that the procedure is complicated and somewhat inefficient. However, procedures continue to improve. Sperm sexing as currently practised is expensive, partly due to inefficiencies, partly due to personnel costs for the many steps, and partly due to the high cost of equipment and its maintenance. Despite these costs and complexities, the procedure works, and already appears to be commercially viable for niche applications in several species. Much simpler equipment designed specifically for sperm sexing probably will become available within a few years. As efficiencies improve and costs decline, sperm sexing will be applied more widely.

The authors gratefully acknowledge the conscientious assistance of Zell Brink, Michael Evans, John Schenk, Sarah Seidel, Tae-Kwang Suh, Lisa Tubman, and Sallie Varner in preparing this review. Reported research was supported financially by XY, Inc., Fort Collins, CO, USA and the Colorado State University Experiment Station.

\section{References}

Key references are identified by asterisks.

*Amann RP and Seidel GE, Jr (Eds) (1982) Prospects for Sexing Mammalian Sperm Colorado Associated University Press, Boulder, CO

Barlow P and Vosa CG (1970) The Y chromosome in human spermatozoa Nature, London 226 961-962

Beal WE, White LM and Garner DL (1984) Sex ratio after insemination of bovine spermatozoa isolated using a bovine serum albumin gradient Journal of Animal Science 58 1432-1436

Blecher SR, Howie R, Li S, Detmar J and Blahut L (1999) A new approach to immunological sexing of sperm Theriogenology 52 1309-1321

Buchanan BR, Seidel GE, Jr, McCue PM, Schenk JL, Herickhoff LA and Squires EL (2000) Insemination of mares with low numbers of either unsexed or sexed spermatozoa Theriogenology 53 1333-1344

Catt SL, Sakkas D, Bizzaro D, Bianchi PG, Maxwell WMC and Evans G (1997) Hoechst staining and exposure to UV laser during flow cytometric sorting does not affect the frequency of detected endogenous DNA nicks in abnormal and normal spermatozoa Molecular Human Reproduction 3 821-825

Doyle SP, Seidel GE, Jr, Schenk JL, Herickhoff LA, Cran D and Green RD (1999) Artificial insemination of lactating Angus cows with sexed semen Proceedings Western Section, American Society of Animal Science $\mathbf{5 0}$ 203-205 
Ericsson RJ and Ericsson SA (1999) Sex ratios. In Encyclopedia of Reproduction Vol. 4 pp 431-437 Eds E Knobil and JD Neill. Academic Press, London

Ericsson RJ, Langevin CN and Nishino M (1973) Isolation of fractions rich in Y spermatozoa Nature 246 421-424

Evans JM, Douglas TA and Renton JP (1975) An attempt to separate fractions rich in human Y sperm Nature 253 352-354

Evenson DP (1989) Flow cytometric analysis of toxic chemical induced alteration in testicular cell kinetics and sperm chromatin structure. In New Trends in Genetic Risk Assessment pp 343-369 Eds G Jolles and A Cordier. Academic Press, New York

Fugger EF (1999) Clinical experience with flow cytometric separation of human $\mathrm{X}$ - and $\mathrm{Y}$-chromosome bearing sperm Theriogenology 52 $1345-1440$

Fugger EF, Black SH, Keyvanfar K and Schulman JD (1998) Births of normal daughters after Microsort spermatozoa separation in intrauterine insemination, IVF or ICSI Human Reproduction 13 2367-2370

*Garner DL (2001) Sex-sorting mammalian sperm: concept to application in animals Journal of Andrology 22 519-526

Garner DL, Schenk JL and Seidel GE, Jr (2001) Chromatin stability in sexsorted sperm. In Andrology in the Twenty-first Century. Proceedings of the VIlth International Congress of Andrology, Montreal, Canada: Short Communications pp 3-7 Eds B Robaire, H Chemes and CR Morales. Medimond, Englewood, NJ

Guthrie HD, Johnson LA, Garrett WM, Welch GR and Dobrinsky JR (2002) Flow cytometric sperm sorting: effects of varying laser power on embryo development in swine Molecular Reproduction and Development 61 87-92

Guyer MF (1910) Accessory chromosomes in man Biological Bulletin of Marine Biology Laboratory, Woods Hole 19 219-234

Hendriksen PJM (1999) Do $X$ and $Y$ spermatozoa differ in proteins? Theriogenology 52 1295-1307

Hendriksen PJM, Welch GR, Grootegoed JA, vander Lende T and Johnson LA (1996) Comparison of detergent-solubilized membrane and soluble proteins from flow cytometrically sorted $\mathrm{X}$ - and $\mathrm{Y}$-chromosome bearing porcine spermatozoa by high resolution 2-D electrophoresis Molecular Reproduction and Development 45 342-450

Herrmann BG, Koschroz B, Wertz K, McLaughlin KJ and Kispert A (1999) A protein kinase encoded by the $t$ complex responder gene causes non-mendelian inheritance Nature 402 141-146

Hoppe PC and Koo GC (1984) Reacting mouse sperm with monoclonal H-Y antibodies does not influence sex ratio of eggs fertilized in vitro. Journal of Reproductive Immunology 6 1-9

Howes EA, Miller NGA, Dolby C, Hutchings A, Butcher GW and Jones R (1997) A search for sex-specific antigens on bovine spermatozoa using immunological and biochemical techniques to compare the protein profiles of $X$ and $Y$ chromosome-bearing sperm populations separated by fluorescence-activated cell sorting Journal of Reproduction and Fertility 110 195-204

Johnson LA (1995) Separation of $X$ and $Y$ chromosome bearing sperm based on DNA differences Reproduction, Fertility and Development 7 893-903

Johnson LA (2000) Sexing mammalian sperm for production of offspring: the state-of-the-art Animal Reproduction Science 60-61 93-107

*Johnson LA and Seidel GE, Jr (Eds) (1999) Current status of sexing mammalian sperm Theriogenology 52 1267-1484

Johnson LA and Welch GR (1999) Sex preselection: high-speed flow cytometric sorting of $X$ and $Y$ sperm for maximum efficiency Theriogenology 52 1323-1341

Johnson LA, Flook JR and Look MV (1987) Flow cytometry of $X$ and $Y$ chromosome-bearing sperm for DNA using an improved preparation method and staining with Hoechst 33342 Gamete Research 17 203-212

*Johnson LA, Flook JP and Hawk HW (1989) Sex preselection in rabbits: live births from $X$ and $Y$ sperm separated by DNA and cell sorting Biology of Reproduction 41 199-203

Johnson LA, Welch GR, Keyvanfar K, Dorfmann A, Fugger EF and Schulman JD (1993) Gender preselection in humans? Flow cytometric separation of $X$ and $Y$ spermatozoa for the prevention of $X$-linked diseases Human Reproduction 8 1733-1739
Johnson LA, Welch GR and Garner DL (1994) Improved flow sorting resolution of $\mathrm{X}$ - and $\mathrm{Y}$-chromosome bearing viable sperm separation using dual staining and dead cell sorting Cytometry 17 Supplement 7 (Abstract 83) 28

Kaneko S, Oshiro S, Kobayashi T, Itzuka R and Mohri H (1984) Human $X$ - and Y-bearing sperm differ in cell surface sialic acid content Biochemical and Biophysical Research Communications 124 950-955

Kiddy CA and Hafs HD (Eds) (1971) Sex Ratio at Birth - Prospects for Control American Society of Animal Science, Savoy, IL

Libbus GL, Perreault SD, Johnson LA and Pinkel D (1987) Incidence of chromosome aberrations in mammalian sperm stained with Hoechst 33342 and UV-laser irradiated during flow sorting Mutation Research $182265-274$

Mohri H, Oshio S, Kaneko S, Kobayashi T and lizuka R (1987) Separation and characterization of mammalian X-and Y-bearing sperm. In New Horizons in Cell Research pp 469-481 Ed. H Mohri. Japan Scientific Society Press, Tokyo/Gordon and Breach Scientific Publishers, New York

Morrell JM and Dresser DW (1989) Offspring from insemination with mammalian sperm stained with Hoechst 33342, either with or without flow cytometry Mutation Research 224 177-189

Ollero M, Perez-Pe R, Gargallo I, Morlanes S, Osada J, Muiño-Blanco T and Cebrian-Perez J (2000) Separation of ram spermatozoa bearing $\mathrm{X}$ and $\mathrm{Y}$ chromosome by centrifugal countercurrent distribution in an aqueous two-phase system Journal of Andrology 21 921-928

Rens W, Welch GR and Johnson LA (1998) A novel nozzle for more efficient sperm orientation to improve sorting efficiency of $\mathrm{X}$ - and $\mathrm{Y}$ chromosome-bearing sperm Cytometry 33 476-481

Rens W, Welch GR and Johnson LA (1999) Improved flow cytometric sorting of X-and Y-chromosome bearing sperm: substantial increase in yield of sexed sperm Molecular Reproduction and Development 52 50-56

Schenk JL, Suh TK, Cran DG and Seidel GE, Jr (1999) Cryopreservation of flow-sorted bovine sperm Theriogenology 52 1375-1391

Seidel GE, Jr (1988) Sexing spermatozoa and embryos. In Proceedings $X I$ International Congress of Animal Reproduction and Artificial Insemination (Dublin) Vol. 5 pp 136-144. University College, Dublin

*Seidel GE, Jr (1999) Sexing mammalian spermatozoa and embryos - state of the art Journal of Reproduction and Fertility Supplement 54 475-485

*Seidel GE, Jr, Schenk JL, Herickhoff LA, Doyle SP, Brink Z, Green RD and Cran DG (1999) Insemination of heifers with sexed spermatozoa Theriogenology 52 1407-1420

Sharpe JC, Schaare PN and Künnemeyer R (1997) Radially symmetric excitation and collection optics for flow cytometric sorting of aspherical cells Cytometry 29 363-370

Suh TK and Schenk JL Pressure during flow sorting of bull sperm affects postthaw motility characteristics Theriogenology $\mathbf{5 9}$ (in press; abstract)

van Munster EB (2002) Interferometry in flow to sort unstained X- and Y-chromosome bearing bull spermatozoa Cytometry 47 192-199

van Munster EB, Stap J, Hoebe R, te Meerman GJ and Aten JA (1999a) Difference in volume of $\mathrm{X}$ - and $\mathrm{Y}$-chromosome bearing bovine sperm heads matches differences in DNA content Cytometry 35 125-128

van Munster EB, Stap J, Hoebe RA, te Meerman GJ and Aten JA (1999b) Difference in sperm head volume as a theoretical basis for sorting X-and Y-bearing spermatozoa: potential and limitations Theriogenology $\mathbf{5 2}$ 1281-1293

Watkins A, Chan PJ and Kalugdan TH (1996) Analysis of the flow cytometer stain Hoechst 33342 on human spermatozoa Molecular Human Reproduction 2 709-712

Welch GR and Johnson LA (1999) Sex preselection: laboratory validation of the sperm sex ratio of flow sorted X- and Y-sperm by sort reanalysis for DNA Theriogenology 52 1343-1352

White IG, Mendoza G and Maxwell WHC (1984) Preselection of sex of lambs by layering spermatozoa on protein columns. In Reproduction in Sheep pp 299-300 Eds DR Lindsay and DT Pearce. Cambridge University Press, Cambridge

Young SD and Hill RP (1989) Radiation sensitivity to tumor cells stained in vitro with the bisbenzimide fluorochrome Hoechst 33342 British Journal of Cancer 60 715-721 